\title{
Reproducible LTE uplink performance analysis using precomputed interference signals
}

\author{
Volker Pauli, Muhammad Danish Nisar ${ }^{*}$ and Eiko Seidel
}

\begin{abstract}
The consideration of realistic uplink inter-cell interference is essential for the overall performance testing of future cellular systems, and in particular for the evaluation of the radio resource management (RRM) algorithms. Most beyond-3G communication systems employ orthogonal multiple access in uplink (SC-FDMA in LTE and OFDMA in WiMAX), and additionally rely on frequency-selective RRM (scheduling) algorithms. This makes the task of accurate modeling of uplink interference both crucial and non-trivial. Traditional methods for its modeling (e.g., via additive white Gaussian noise interference sources) are therefore proving to be ineffective to realistically model the uplink interference in the next generation cellular systems.

In this article, we propose the use of realistic precomputed interference patterns for LTE uplink performance analysis and testing. The interference patterns are generated via an LTE system-level simulator for a given set of scenario parameters, such as cell configuration, user configurations, and traffic models. The generated interference patterns (some of which are made publicly available) can be employed to benchmark the performance of any LTE uplink system in both lab simulations and field trials for practical deployments. It is worth mentioning that the proposed approach can also be extended to other cellular communication systems employing OFDMA-like multiple access with frequency-selective RRM techniques.

The proposed approach offers twofold advantages. First, it allows for repeatability and reproducibility of the performance analysis. This is of crucial significance not only for researchers and developers to analyze the behavior and performance of their systems, but also for the network operators to compare the performance of competing system vendors. Second, the proposed testing mechanism evades the need for deployment of multiple cells (with multiple active users in each) to achieve realistic field trials, thereby resulting in significant cost (and time) savings in the field trails.
\end{abstract}

\section{Introduction}

A crucial phase, before the successful deployment of a technology is its testing and validation under realistic operating conditions. To this end, tests are typically designed and conducted in lab as well as in actual deployment scenarios. Tests conducted in a lab environment allow for a quick and cost-efficient evaluation of various algorithms and techniques. However, it is hard to mimic the real-life environment in a lab. On the other hand, tests conducted on actual deployment scenariosoften referred to as field trials-allow for an exact emulation of real-life scenarios, in which a system is expected to operate. On the downside, field trials are generally time consuming and expensive.
In terms of the mode of evaluation, the testing mechanisms can be grouped into two categories, namely, conformance and performance testings. Conformance testing refers to a sequence of pass/fail tests laid down typically by the standardization body itself, to certify the developed products being compliant or non-compliant with the various modes of operation in the given standard [1,2].

Performance testing-often referred to as 'beyond conformance testing' [3]-is an extension of conformance testing to a more sophisticated quantitative evaluation of commercial products. This allows network operators to compare different products from different vendors against each other. Although, the purpose of all the testing methodologies is different, all share some common desirable features.

\footnotetext{
* Correspondence: mdanishnisar@ieee.org

Eiko Seidel Nomor Research GmbH, Munich, Germany
}

\section{Springer}

(C) 2011 Pauli and Nisar; licensee Springer. This is an Open Access article distributed under the terms of the Creative Commons Attribution License (http://creativecommons.org/licenses/by/2.0), which permits unrestricted use, distribution, and reproduction in any medium, provided the original work is properly cited. 
- Realistic: The environment used for testing should mimic the actual network deployments as closely as possible. This is of significance for the relevance of both conformance and especially performance test results. In terms of closeness to reality, field tests enjoy an edge over lab testing where the real-life conditions are only artificially modeled.

- Reproducible: It is highly desirous of all the testing methodologies to be reproducible. This allows for repeatability of tests at a later time, and also to compare the performance of various competing techniques over an exact same set of conditions. While lab tests can be made reproducible, field trials, in part, suffer from non-reproducibility because of the randomness effects that are inherent in the environment. - Time and cost efficient: All the testing methodologies are required to be time efficient and of low cost, but this often conflicts with the requirement of being realistic. For instance, field testing, while offering the most realistic test environment, has the drawback of being most expensive and time consuming.

In this article, we propose a novel testing mechanism that combines the desirable features of being realistic and reproducible, besides being time and cost efficient. The proposed mechanism can be effectively applied to lab testing as well as field trials, and not only for conformance testing, but also more importantly perhaps for the performance testing. The approach can be used by developers (vendors) to debug and benchmark the performance of their alternative algorithms, and also by network operators in arriving at better technically informed decisions about the selection from multiple vendors.

Although the proposed testing mechanism is generic and applicable to a broad set of scenarios and standards, in this article, we discuss it in the context of LTE uplink inter-cell interference modeling. Inter-cell interference is a major bottleneck in the performance of current and future cellular systems, which are gradually pushing toward diminishing frequency reuse factors and dense deployments. In LTE, the consideration of realistic uplink inter-cell interference is essential for testing radio resource management (RRM) algorithms as well as for overall performance testing. Traditional methods for interference modeling (e.g., via additive white Gaussian noise (AWGN) interference sources) are no longer able to realistically model the uplink interference, especially in the orthogonal uplink scenarios employing frequency-selective RRM algorithms as in the SC-FDMA-based multiple access in LTE uplink $[4,5]$.

We employ a reconfigurable system-level LTE simulator to model the typical deployment scenarios outlined in the 3GPP specifications and implementation guidelines $[6,7]$. Depending on the various system parameters, the behavior of uplink inter-cell interference from neighboring cells is recorded in trace files, and a postprocessing of these simulator trace files leads to realistic time-domain uplink interference signals for the given system settings. These precomputed uplink interference signals can be employed in a variety of ways.

- Simulations: System-level simulators with only isolated cell capabilities can benefit from the generated interference signals by adding to it the interferencefree uplink-received signal, thereby effectively modeling a multi-cellular scenario.

- Lab testing: In lab testing environments, it has been quite difficult (if not impossible) to accurately model a multi-cellular deployment. The precomputed interference signals offer the possibility to realistically model the uplink interference, and thus provide a more realistic test environment for LTE uplink, even in a lab.

- Field testing: Deploying a multi-cellular system, with multiple users active in each cell, is an expensive, non-flexible exercise. The precomputed interference signals, in combination with commercially available vector signal generators (VSGs), can be used to effectively create a virtual multi-cell interference environment without the need of actually deploying multiple cells (see the illustration in Figure 1). In addition, the proposed approach offers the advantage of reproducible interference signals which can be used to compare the performance of competing algorithms or products in field trials.

The remainder of this article is organized as follows: Section 2 describes the system-level simulator employed to obtain the interference power patterns. In Section 3, the approach used for converting these into time-domain interference signal is presented. Section 4 presents the details of scenarios used for the generation of the publicly available LTE uplink interference signal files. Section 5 contains some simulation results to illustrate the importance of using realistic interference modeling for LTE uplink performance analysis. Section 6 outlines the usage of the proposed interference patterns, and Section 7 concludes this article.

\section{System-level simulator for LTE networks}

Figure 2 illustrates the structure of the system-level simulator employed in this study. Apart from a graphical user interface that is not shown here, it consists of three major entities: the network simulator itself, a channel model block, and a block for modeling the linklevel transmission errors.

The network simulator is based on a comprehensive multi-cellular simulation tool for both the uplink and downlink of an LTE system, and contains the traffic 


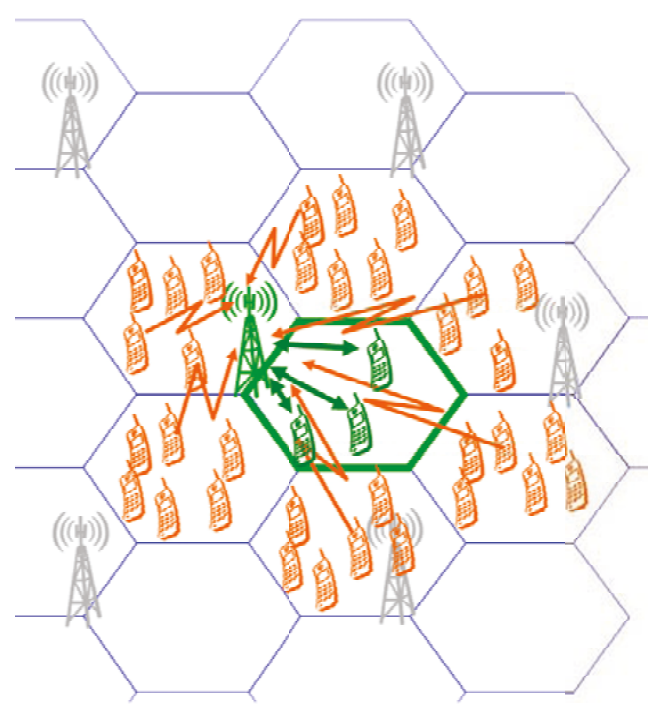

(a) Traditional approach: deploying multiple cells with active users in each cell.

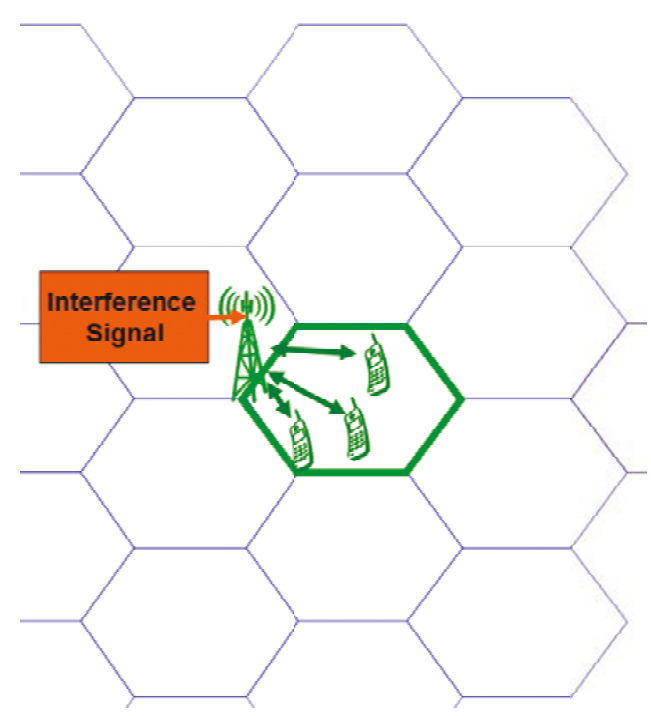

(b) Proposed approach: employing precomputed interference patterns to virtually create a multi-cell interference environment.

Figure 1 Illustration of cost/complexity/time savings of the proposed approach to uplink performance analysis. (a) Traditional approach deploying multiple cells with active users in each cell. (b) Proposed approach: employing pre-computed interference patterns to virtually create a multi-cell interference environment.

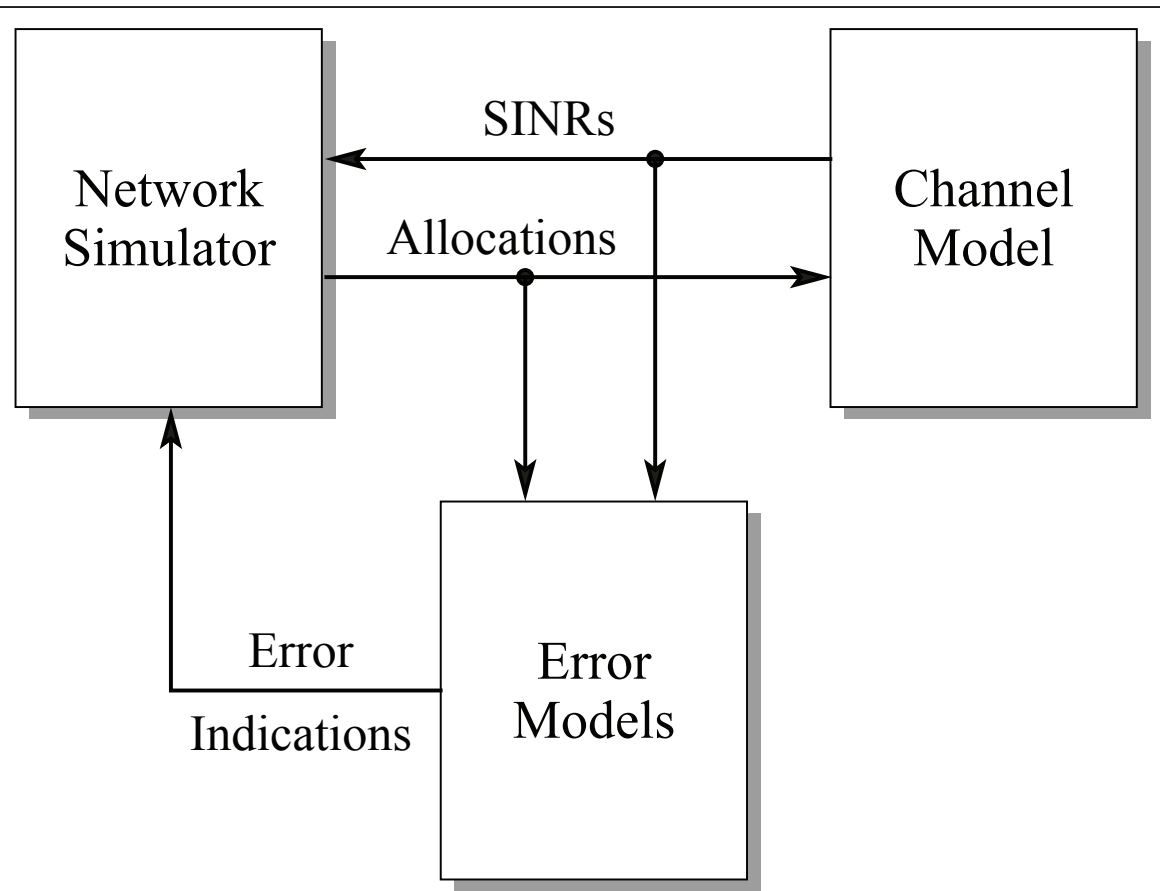

Figure 2 Structure of the system-level simulator for LTE networks 
sources and protocol stacks of different network elements. With regard to this study, the simulator models the traffic sources at each user equipment (UE), ensures that appropriate time and frequency resources are assigned to them via realistic scheduling algorithms at each enhanced-nodeB (eNB) and takes into account the realistic availability of information, e.g., regarding channel state, buffer status, etc.

The network simulator sends information about the scheduled uplink and downlink transmissions of different cells simulated to the channel model block. The latter uses these to compute signal-to-interference and noise ratios (SINR) for the different links between base stations and UEs, taking into account the geographical information about the network, path-loss, shadowing, fast fading, inter-cell interference, etc. The SINRs are computed at a resolution of one sample per physical resource block (PRB) and per transmission time interval (TTI). These SINRs are passed back to both the network simulator and the error models. The network simulator uses them to, e.g., compute channel quality indicator information at the UEs, or extract uplink channel state information at the base station based on some configured channel sounding mode or simply in-band pilots.

The error models' block ${ }^{\mathrm{a}}$ accepts the SINRs computed by the channel model at PRB resolution and the allocation information sent out by the network simulator. Using the so-called mutual information effective SINR metric method [8], it computes effective SINRs for each active transmission link in the whole network. The effective SINR is then used in conjunction with the precomputed link-level results for the AWGN channel to determine the error indications for the individual transmissions. This efficient link-to-system level modeling [9] allows us to bypass the actual link-level processing such as channel en-/decoding, (de-)modulation, layer (de-) mapping, precoding, and equalization, with minimal complexity during system-level simulations.

\section{Post-processing of simulator output}

The system-level simulator as a whole has the capability to write various quantities that appear in the simulation to trace files for offline evaluation. This ranges from throughput or delay at packet data convergence protocol (PDCP) layer, down to uplink and downlink SINRs at PRB resolution, and in particular-as used in this studythe aggregated uplink inter-cell interference power as received by the base station at the center of the simulated network. The aggregated uplink interference power computed by taking into account the geographical information about the network, path-loss, shadowing, fast fading, antenna gains, etc., is stored at a resolution of one sample per PRB along the frequency direction, and per TTI along the time direction.

It shall be noted here that the system-level simulator, used for the generation of the uplink interference power trace, does not make any assumptions on the synchronism between interfering users. Thus, the interference powers and the resulting aggregate time-domain interference signal represent a scenario where the interfering signals arrive from different users with arbitrary (nonidentical) delays, as in a real-life deployment.

We post-process the trace files associated with the uplink interference power to generate realistic interference signals in time domain. The process is depicted by the flow chart in Figure 3. In the first step, the interference trace file containing the uplink interference powers is used to generate a random frequency-domain DFTSpread-OFDM signal $[10,11]$ with the appropriate power levels for each PRB over each TTI. This is achieved by increasing the resolution of the interference power from per PRB to per sub-carrier via interpolation. Then, either a Gaussian random signal or a random DFT-SpreadOFDM LTE uplink signal, of length corresponding to one TTI, is generated for each sub-carrier with the given power level. The process is repeated for each TTI.

In the second step, the frequency-domain signal is transformed to the time-domain signal using the typical OFDM processing. This involves carrying out an IFFT of the samples across the various sub-carriers of an OFDM block, and then inserting a cyclic prefix of appropriate duration. The signal is then parallel-to-serial converted for each OFDM block, leading finally to the realistic time-domain uplink interference signal which would be experienced by an uplink receiver in a multi-cell deployment with the given system settings.

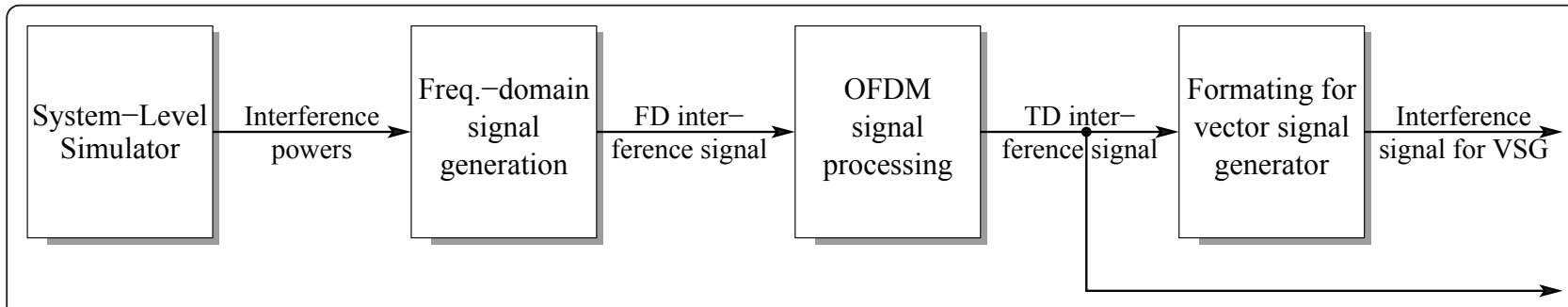

Figure 3 Flow chart illustrating the post-processing to obtain interference signal from trace file of the system-level simulator. 
As a last step, the interference signal may additionally be transformed into the proprietary formats of the commonly available commercial VSGs, as explained in Section 6 . To accurately model the uplink interference, the resulting VSG signal is supposed to be coupled into the path between the antenna and the receiver (e.g., MRC) with prescribed power settings.

\section{Interference scenarios under consideration}

For the generation of the different uplink interference patterns, we considered a homogeneous LTE uplink deployment with multiple cells. The parameters for system layout and simulation scenarios have been adopted from [[6], Appendix A]. These are, however, mostly identical to those found in other 3GPP specifications like [7], and in the NGMN evaluation scenarios [12]. Although many more system configurations are conceivable, the adopted parameters have been defined as being representative of future LTE networks by the industry and are being widely used in research and standardization.

\subsection{Cell layout}

Table 1 lists the main parameters affiliated with the eNB, the UE, and those that characterize the cell layout and system configuration. As mentioned above, the parameters are based on the 3GPP prescribed system deployments as outlined in [[6], Appendix A].

Table 1 System configuration parameters

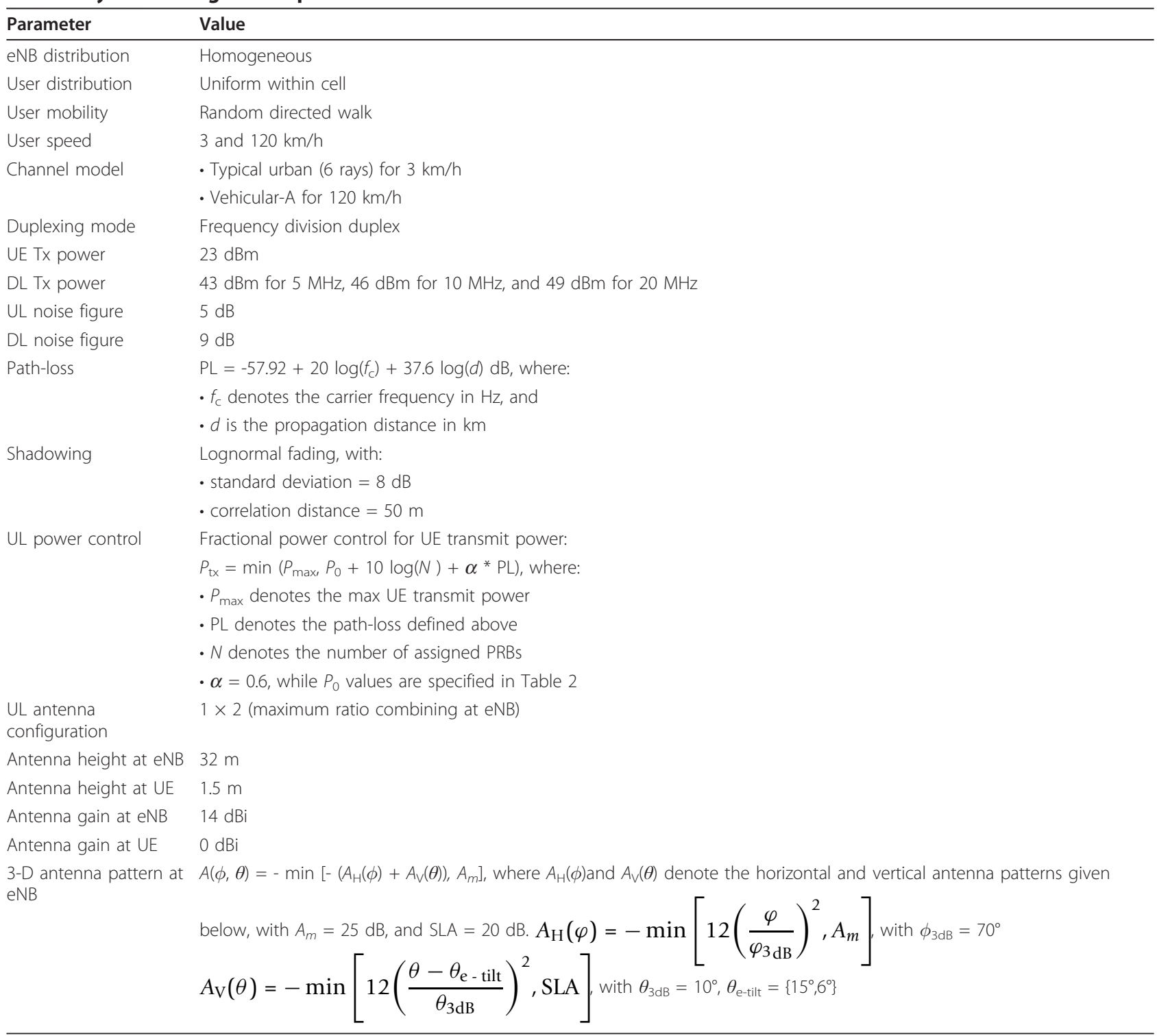




\subsection{Traffic types}

For the simulation results in this article, we consider two fundamentally different traffic models for the uplink user traffic. As will be shown later, these have a profound impact on the characteristics of the resulting interference patterns.

\section{- Full buffer traffic}

- Full transmit buffers.

- Scheduling: In time domain, all the active UEs are scheduled in each TTI. In frequency domain, proportional fair scheduling [[11], Chapter 7] with periodic wideband channel sounding is employed, with 5 PRBs per UE per TTI.

- Number of UEs in radio resource control (RRC)connected state depends upon the configured cell load and the system bandwidth. For instance, 70\% load in a $10-\mathrm{MHz}$ system (with $50 \mathrm{PRBs}$ ) is obtained via seven active UEs each being assigned five PRBs in every TTI.

\section{- VoIP traffic}

- VoIP calls, $14 \mathrm{kbps}$, real-time transport protocol.

- Scheduling: In order to avoid limitations from the associated control signaling, we aggregate a minimum of two VoIP packets for each UE before it is eligible for scheduling. In time domain, a round robin scheduler is employed, which lets a maximum of 10 UEs to be assigned resources by the frequency-domain scheduler in the current TTI, with preference being given to UEs with retransmissions. In frequency domain, proportional fair scheduling with adaptive transmission bandwidth (e.g., [13]) is employed for the 10 UEs assigned in each TTI.

- Number of UEs in RRC-connected state depends upon the configured cell load and the system bandwidth. The maximum number of supportable VoIP calls is determined empirically for each scenario. A VoIP user is in outage if more than $2 \%$ of its packets in a 8-s call cannot be correctly received within $80 \mathrm{~ms}$. The system capacity is defined as the maximum load in which more than $95 \%$ of the users are not in outage (see, for instance, [[14], Appendix A.2]). Lower cell loads, such as 30 and $70 \%$, correspond, respectively, to 30 and $70 \%$ of the maximum number of supportable UEs.

\subsection{Selected scenarios}

Based on the reference system deployments recommended in [[6], Appendix A], three system layouts are selected for the generation of LTE uplink interference signals. These scenarios, along with their associated parameters, are specified in Table 2, where $f_{\mathrm{c}}$ denotes the carrier frequency, and $P_{O}$ is the power control parameter, as introduced in Table 1. Each of the three interference scenarios defined in the table is configured with

- one of the two traffic types (full buffer and VoIP), - a prescribed load level (30 and 70\% for 3GPP-C1 and ITU-HS, while 70 and 100\% for 3GPP-C3), and - one of the three system bandwidths $(5,10$, and 20 $\mathrm{MHz}$.

Thus, in total, there are 36 uplink interference scenarios with different parameter combinations. These selected interference scenarios are partitioned into standard and extended uplink interference packages. The standard package consisting of 3GPP-C1 with 70\% load is publicly available free of charge under a temporary evaluation license that can be obtained via [15].

\section{Simulation results}

\subsection{Snapshots of interference patterns}

In this sub-section, we discuss the nature and characteristics of LTE uplink interference via two sample interference snapshots below.

\subsubsection{Full buffer scenario}

Figure 4 shows a sample interference snapshot for the 3GPP-C1 scenario with full buffer traffic, at a 70\% load level, for a $10-\mathrm{MHz}$ system. The snapshot shows interference power at TTI and PRB resolution, for a time interval of $50 \mathrm{~ms}(50 \mathrm{TTI})$ and across the whole $10-\mathrm{MHz}$ bandwidth (50 PRBs).

For the full buffer traffic, there is a limited number of high-rate users (seven active users in this scenario) configured in each cell. Since the configured cell load is $70 \%$ for all the cells in the scenario, it can happen by chance that no user in the direct neighboring cells is scheduled on a particular PRB, leading to a very low interference level on that PRB. On the other hand, a high interference power is experienced on PRBs, where a neighboring cell schedules a UE which is close to the cell edge of the cell of interest. Thus, we observe strong variations in the inter-cell interference power across PRBs. The interference is dominated

Table 2 Interference scenarios under consideration

\begin{tabular}{lllllll}
\hline Name & ISD $(\mathbf{m})$ & $\boldsymbol{f}_{\mathbf{c}}(\mathrm{GHz})$ & UE speed $(\mathbf{k m} / \mathbf{h})$ & Penetration loss $(\mathrm{dB})$ & $\boldsymbol{P}_{\mathbf{0}}$ (full buffer) $(\mathbf{d B m})$ & $\boldsymbol{P}_{\mathbf{0}}(\mathrm{VolP})(\mathrm{dBm})$ \\
\hline 3GPP-C1 & 500 & 2 & 3 & 20 & -60 & -60 \\
3GPP-C3 & 1732 & 2 & 3 & 20 & -69 & -64 \\
ITU-HS & 1732 & 0.8 & 120 & 9 & -57 & -58 \\
\hline
\end{tabular}




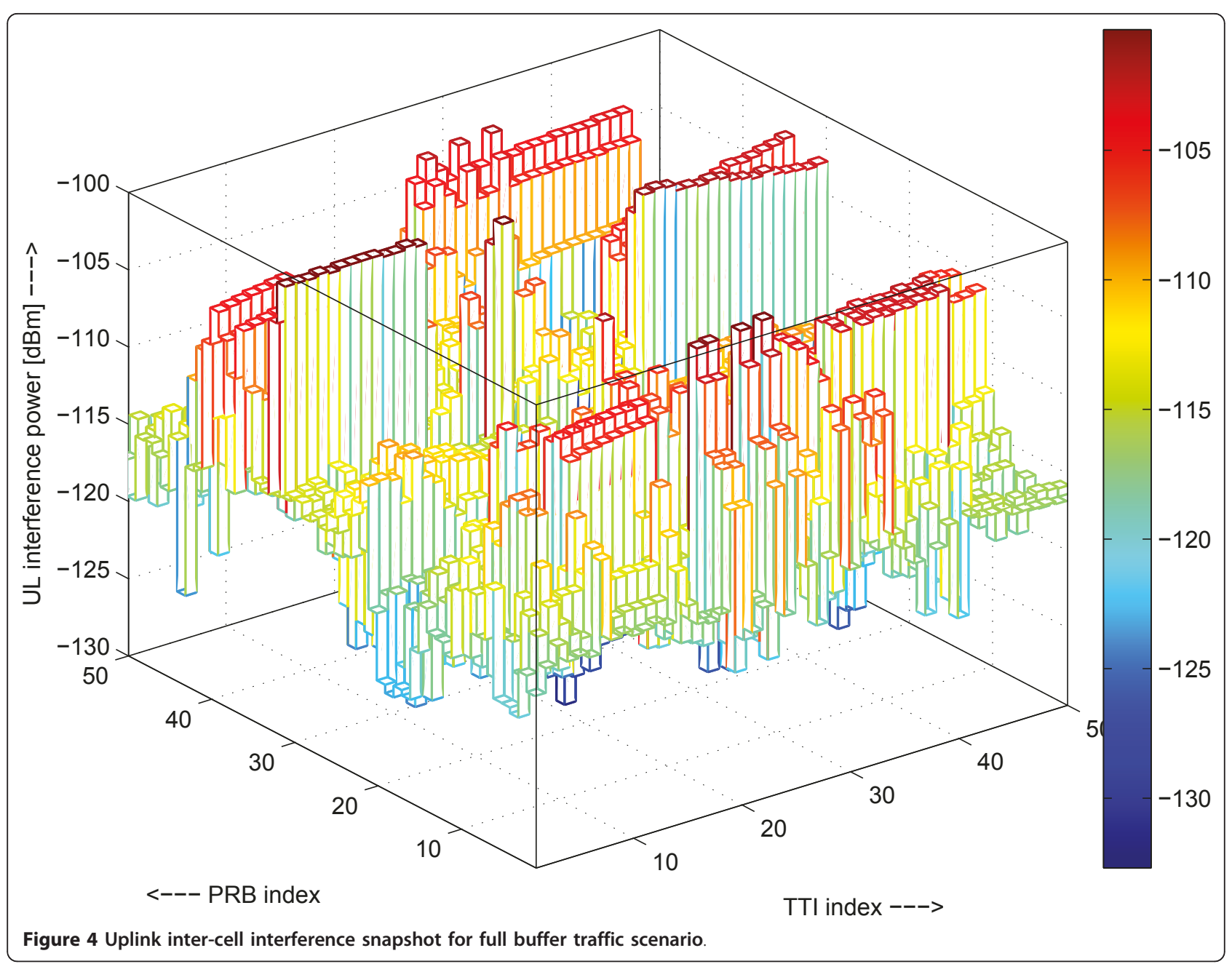

by few interferers or even a single interferer located at the cell edge of the cell under consideration.

Although different users might be scheduled on a particular PRB from one TTI to the next, causing strong interference variations along time as well, users might also continuously be scheduled on the same PRBs for consecutive TTIs. If the latter happens, for a dominating interferer, then we observe a slowly changing interference power level along TTIs, caused only by the user mobility and fast fading.

Overall, it can easily be observed that the inter-cell interference power is far from being flat over time and frequency, and therefore a precise modeling of uplink interference has a significant impact on the evaluation of system performance.

\subsubsection{VolP scenario}

Figure 5 shows a similar interference snapshot for the same scenario except that the user traffic is VoIP instead of full buffer traffic. Because the characteristics of the traffic are fundamentally different from those in the previous scenario, so is the scheduling behavior. For example, UEs only periodically have small amounts of data to transmit. Hence, consecutive allocations of PRBs to the same UE over several consecutive TTIs occur only very rarely. Second, for efficient scheduling of VoIP traffic obeying constraints from availability of downlink control signaling resources, it is typical to use packet aggregation (e.g., [16]), i.e., to accumulate data of a user over, e.g., $40 \mathrm{~ms}$ and assign larger numbers of PRBs simultaneously, but less frequently. In consequence, the uplink interference in Figure 5 exhibits very strong fluctuations from one TTI to the next; however, it shows some correlation in the frequency direction.

Thus, we note that the VoIP and full buffer traffic scenario possess quite different characteristics for the interference pattern and its variations across time and frequency. A precise modeling of uplink interference is therefore crucially needed for an accurate and a reliable performance analysis of cellular systems. This aspect is further highlighted in the next sub-section. 


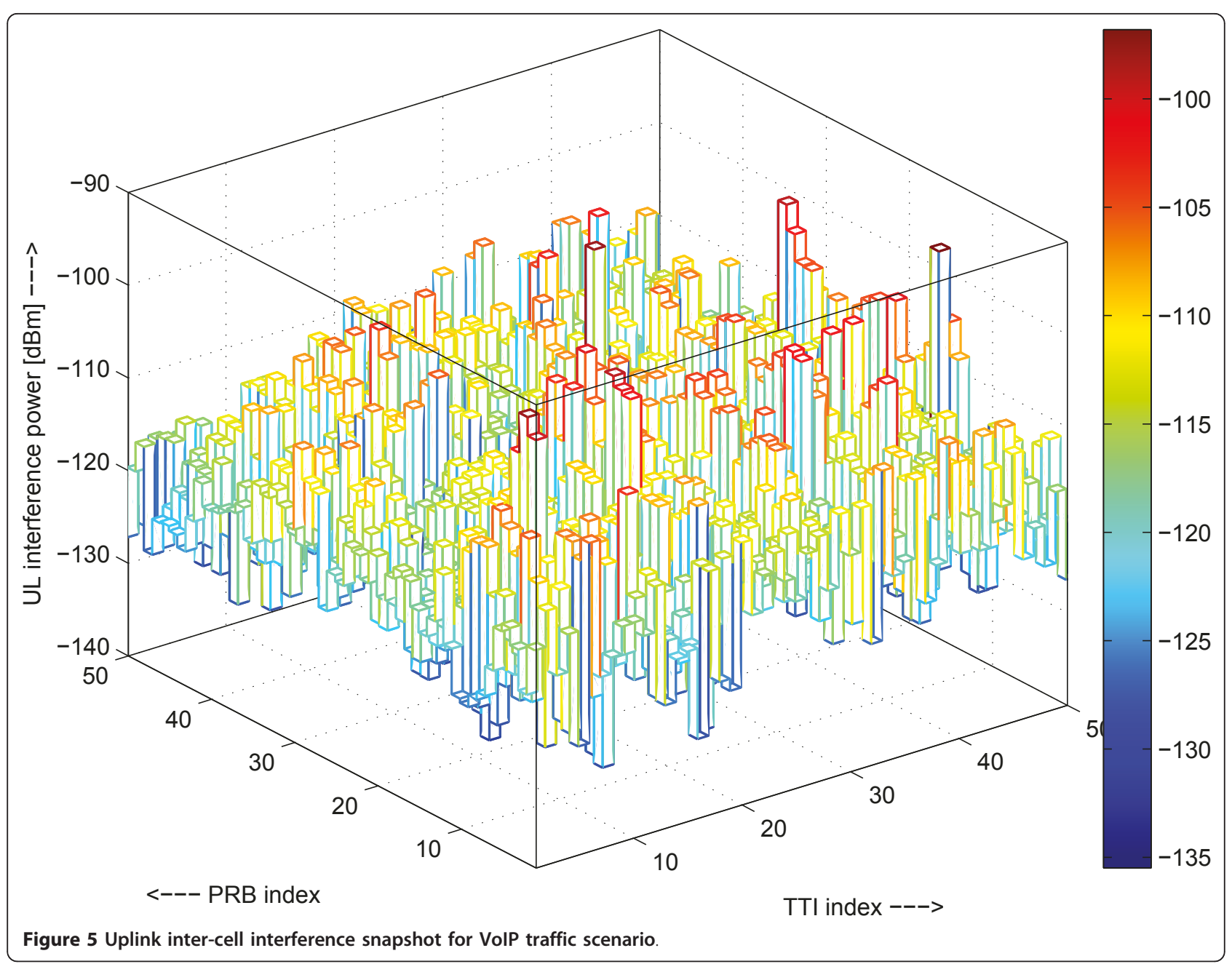

\subsection{Performance comparison between proposed and AWGN interference modeling}

In this sub-section, we study the effect of the proposed and the AWGN-based interference modeling approaches on the system-level performance of an LTE uplink system. To this end, we perform two simulation runs:

- First, we run the simulator with the proposed realistic uplink interference modeling, and measure some relevant performance metrics.

- For the second run, we average (over time and frequency) the per-PRB powers of the uplink interference used in the first run of the simulator, add this average to the per-PRB noise power, and disable the realistic interference. Otherwise, the simulator is configured identically, including the seeds for random number generators. Hence, the total noise and interference power is the same as before, but in the second run, pure AWGN with constant power across time and frequency is present as the sole source of interference.
In the following, we present the comparison for two different traffic scenarios.

\subsubsection{Full buffer scenario}

We consider a full buffer traffic scenario with a load of $70 \%$ in the 3GPP-C1 scenario, described in Section 4, and measure the average UE throughput taking into account the proposed or the AWGN interference model.

Figure 6 shows in terms of throughput, the error made when using AWGN as sole source of interference compared to the case of realistic interference. It can be observed that the user throughput is, in general, overestimated when simulating with AWGN interference. The actual deviation varies significantly depending on the particular position of the UE, showing smaller deviations for UEs in favorable positions and deviations as large as 58\% for cell-edge UEs. The reason is that the fluctuations of realistic interference force the scheduler to select the modulation and coding scheme more conservatively to meet the required target of reliability (herein assumed to be $10 \%$ error probability after the first HARQ transmission). At higher error rate targets, this is less critical, and 


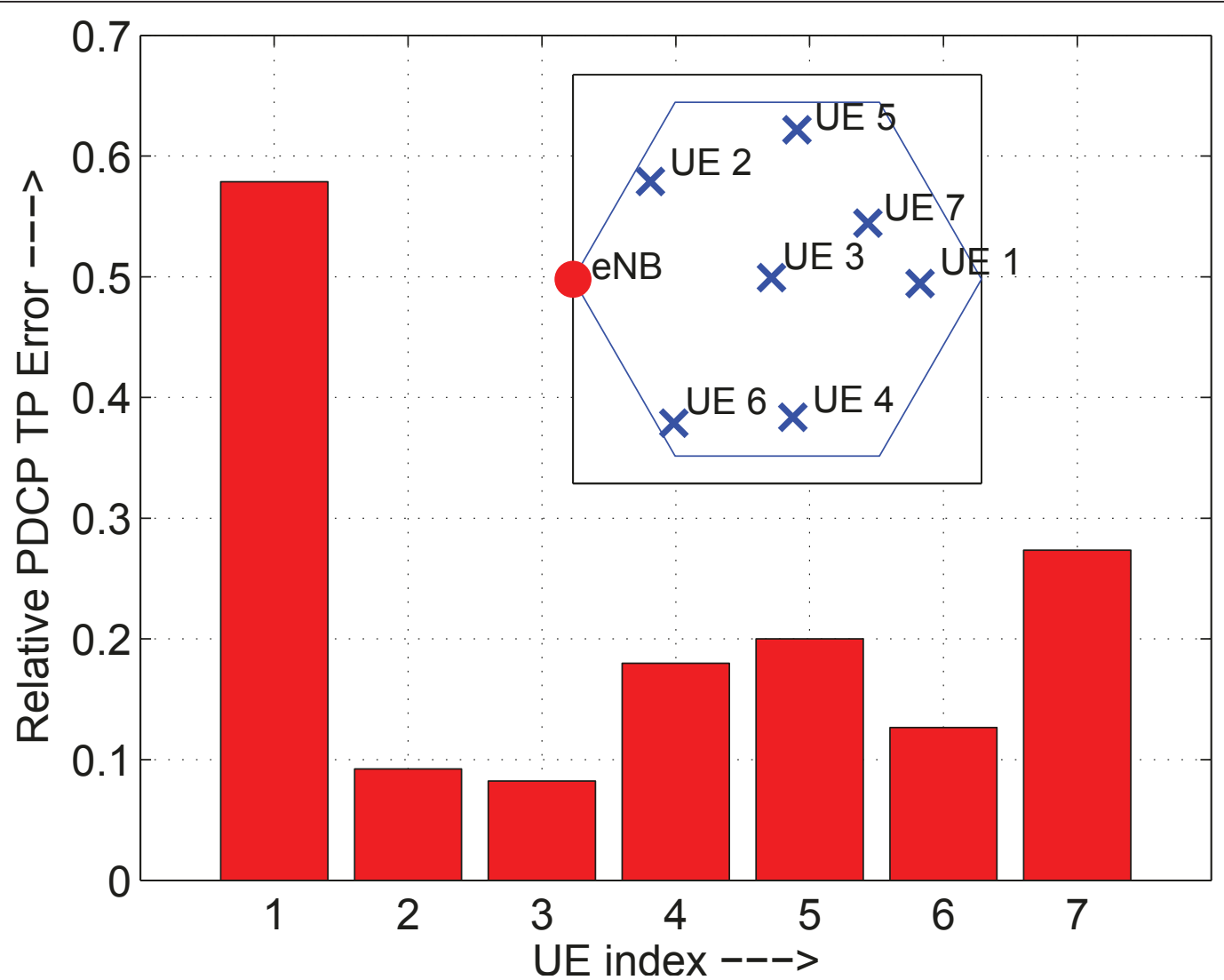

Figure 6 Relative error in user throughput at PDCP layer in the 3GPP-C1 scenario with a 10-MHz system bandwidth, and 70\% load for full buffer traffic.

a channel-aware scheduler can even benefit from the at times slowly changing interference levels (cf. Figure 4), and can therefore make more qualified scheduling decisions leading to a higher throughput. From the illustration of the UE positions as shown in Figure 6, it can be observed that there is a relation between distance from the eNB and the relative throughput error. It is worth mentioning though that this dependence of the relative error on the distance from the eNB becomes less evident, once shadowing (not considered here) comes into play.

\subsubsection{VolP scenario}

In this context, we evaluate the system performance for a VoIP scenario in a manner similar to the one described above, i.e., first with a realistic inter-cell interference and then with AWGN noise of equal average power. Figure 7 shows the performance in terms of the fraction of the satisfied UEs for the two simulation runs. A VoIP UE is said to be satisfied, if it is not in outage that is defined as the state in which more than $2 \%$ of its packets in a 8-s call cannot be correctly received within 80 ms [[14], Appendix A.2].

We observe in Figure 7 that the performance with averaged interference modeling is too optimistic as compared to the performance with realistic interference modeling. This is primarily the result of the extreme fluctuations of interference powers across TTIs that can potentially lead to an increased PDCP delay due to more conservative TFC selections and/or higher percentage of retransmissions.

In a nutshell, comparing the findings from the results presented in Figures 6 and 7, we conclude that there are significant performance deviations once we switch to realistic inter-cell interference modeling, with a tendency to overestimation when replacing realistic interference with simplified AWGN interference of the same power. Furthermore, the dependence of the performance deviations on scenarios under consideration, traffic types, and UE locations, makes it clear that there is no simple rule of thumb to draw conclusions from the results obtained via simplified interference modeling to real system performance.

\section{Usage of interference patterns}

As depicted in Figure 3, the interference signal that we generate can be used primarily in two different ways. First, the generic interference signal (lower output in 


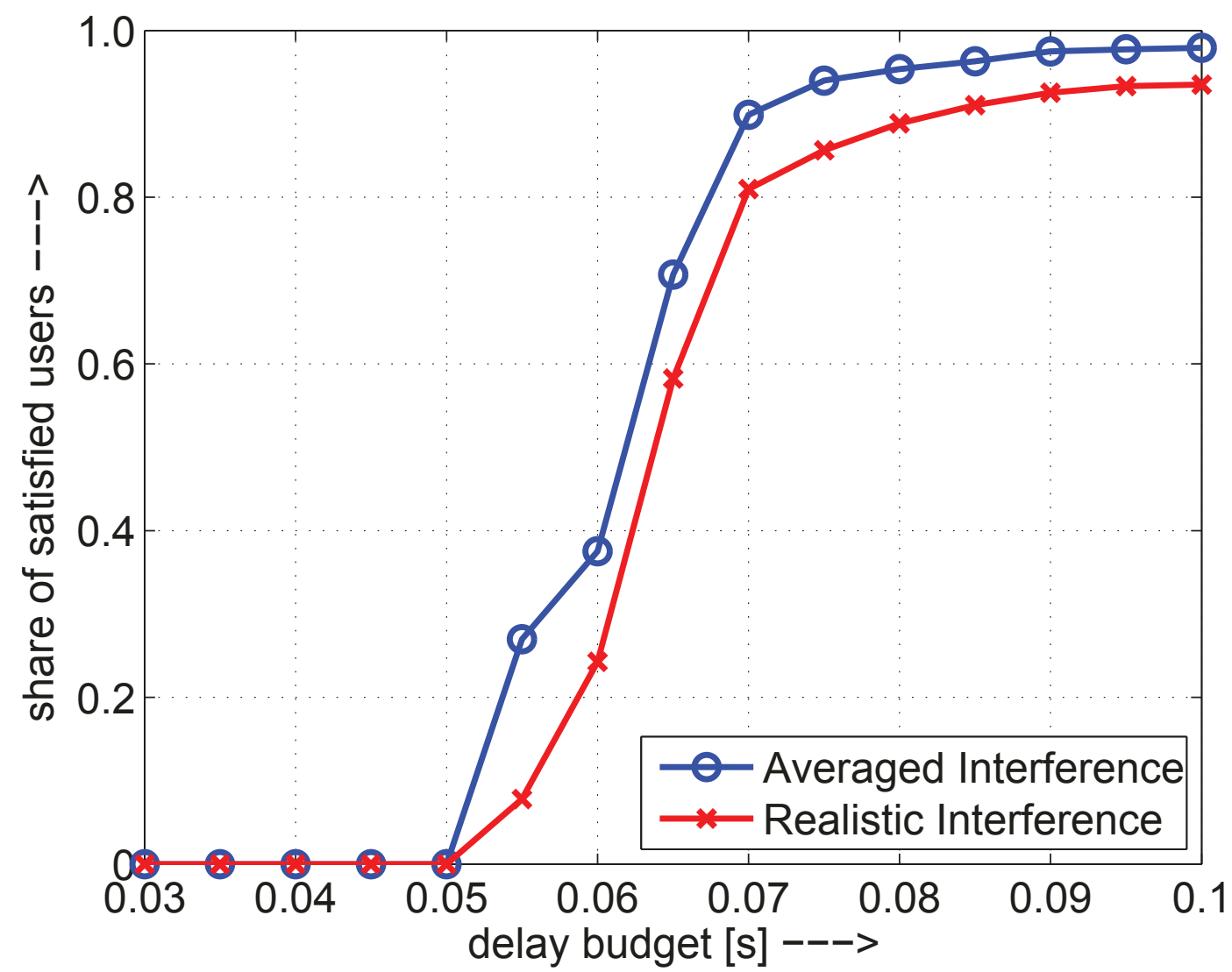

Figure 7 Comparison of VolP user satisfaction in 3GPP-C1 scenario with a 10-MHz system bandwidth and $100 \%$ load.

Figure 3) can directly be used by single-cell simulators for the performance evaluations of various alternative algorithms in a multi-cellular environment. This could be useful for researchers and developers of algorithms to investigate, at an early stage, and/or with relatively small budget, the performance of their algorithms in a realistic multi-cellular environment. Furthermore, as discussed earlier, the precomputed interference files offer the feature of reproducibility. Thus, the tests are completely repeatable and hence useful for debugging as well as benchmarking purposes.

Second, the signal can be transformed into proprietary formats of commercially available signal generators (upper output in Figure 3). Until now, we have collaborated with Agilent Technologies and Rohde \& Schwarz to generate files that are suitable for playback on their commercially available VSGs. We have described the relatively straight-forward playback procedures for the Agilent and R\&S VSGs in the respective user manuals $[17,18]$. Using the signal generators, the generated interference signals can be fed directly into antenna ports of the bases station under test, to effectively emulate a multi-cellular interference environment in the lab or in the field trials (cf. Figure 1). Thus, the proposed mechanism adds the feature of realistic multi-cellular environment emulation to lab and field testings in a cost- and time-efficient manner. The additional feature of reproducibility implies that the tests are completely repeatable, and can be effectively used by network operators to make technically informed decisions and selections between the products of competing vendors using their own performance criteria.

The need for realistic and repeatable performance tests for LTE was felt by the Global Certification Forum which required 3GPP to consider the standardization of realistic end-to-end data performance measurement tests, where the measurement procedures are capable of running under repeatable set of conditions [19]. In compliance of this, the 3GPP technical sub-group, responsible for conformance testing, is currently discussing the terms of reference and a study item, as part of the 3GPP Release 11 specification about UE application layer data throughput performance, was recently agreed in a 3GPP RAN plenary meeting [20]. The proposed testing mechanism is therefore a valued and timely contribution to the entire LTE community. While some of the interference files associated with the standard scenarios, as described in Section 4, are publicly available 
free of charge [15], we have been in collaboration with leading infrastructure vendors who are using the proposed inter-cell interference modeling mechanism in their global LTE field trial activities.

\section{Conclusions}

In this article, we propose a cost- and time-efficient mechanism to realistically model uplink inter-cell interference for an LTE-like orthogonal multiple access and frequency-selective RRM-based cellular system. The proposed approach offers the advantage of reproducibility and repeatability of lab tests and field trials. Thus, it is beneficial for researchers and developers to benchmark the performance of their algorithms over a realistic and reproducible set of conditions. Furthermore, the proposed testing methodology provides network operators an opportunity to make technically informed decisions about the selection of products from their competing system vendors.

\section{Endnote}

${ }^{\text {a }}$ There is one error model for each link between a UE and the respective serving base station.

\footnotetext{
Abbreviations

AWGN: additive white Gaussian noise; eNB: enhanced-nodeB; PDCP: packet data convergence protocol; PRB: physical resource block; RRC: radio resource control; RRM: radio resource management; SINR: signal to interference and noise ratio; TTl: transmission time interval; UE: user equipment.
}

\section{Competing interests}

The authors declare that they have no competing interests.

Received: 28 January 2011 Accepted: 18 September 2011

Published: 18 September 2011

\section{References}

1. 3GPP TS 36.141 (2010-08), Evolved Universal Terrestrial Radio Access (EUTRA); Base Station (BS) Conformance Testing, (Release 10), in Technical Specification Group Radio Access Network (2010)

2. 3GPP TS 36.521 (2010-08), Evolved Universal Terrestrial Radio Access (EUTRA); User Equipment (UE) Conformance Specification, Radio Transmission and Reception, Part 1: Conformance Testing, (Release 9), in Technical Specification Group Radio Access Network (2010)

3. Electrobit, Beyond Conformance Testing in 3GPP LTE. (June 2009) White paper

4. R4-093766 3GPP Tdoc, Analysis of non-Gaussian Inter-Cell Interference Impact on Performance, Agilent Technologies and Nomor Research GmbH, in 3GPP TSG-RAN WG4 \#52bis meeting, Japan (October 2009)

5. R4-103235 3GPP Tdoc, LTE Receiver Performance using AMC with Realistic Noise, Agilent Technologies and Nomor Research GmbH, in 3GPP TSG-RAN WG4 \#56 meeting, Japan (August 2010)

6. 3GPP TR 36.814 V9.0.0 (2010-03), Technical Specification Group Radio Access Network; Evolved Universal Terrestrial Radio Access (E-UTRA); Further advancements for E-UTRA physical layer aspects, (Release 9), in Technical Report 3GPP (2010)

7. 3GPP TR 25.814 V7.1.0 (2009-06), Technical Specification Group Radio Access Network; Physical Layer Aspects for Evolved Universal Terrestrial Radio Access (UTRA), (Release 7), in Technical Report 3GPP (2009)

8. K Brüninghaus, D Astély, T Sälzer, S Visuri, Link Performance Models for System Level Simulations of Broadband Radio Acces Systems, in PIMRC, Berlin, Germany (September 2005)
9. V Pauli, I Viering, C Buchner, E Saad, G Liebl, A Klein, Efficient Link-to-System level Modeling for Accurate Simulations of MIMO-OFDM Systems, in International Conference on Communications, Dresden (June 2009)

10. D Galda, H Rohling, A Low Complexity Transmitter Structure for OFDMFDMA Uplink Systems, in IEEE Vehicular Technology Conference, pp. 1737-1741 (2002)

11. E Dahlman, in $3 G$ evolution: HSPA and LTE for mobile broadband, (Academic Press, 2008)

12. NGMN White Paper, NGMN Radio Access Performance Evaluation Methodology. http://www.ngmn.org (2008)

13. FD Calabrese, in Scheduling and Link Adaptation for Uplink SC-FDMA Systems: A LTE Case Study, Dissertation, Aalborg University, Denmark (April 2009)

14. S4 (10)0858 3GPP Tdoc, Source-Controlled Variable Bit Rate Operation in EVS, Qualcomm Incorporated, AT\&T, in 3GPP TSG-SA4 \#61 meeting (November 2010)

15. Nomor Research GmbH, Contact Form for LTE Uplink Interference Files. http://www.nomor.de/lte-ul-if (2011)

16. H Holma, J Kallio, M Kuusela, P Lundn, E Malkamki, J Ojala, H Wang, Voice over IP (VoIP), in LTE for UMTS: OFDMA and SC-FDMA Based Radio Access, ed. by Holma H, Toskala A (John Wiley \& Sons, Ltd, Chichester, UK, 2009)

17. Nomor Research $\mathrm{GmbH}$ and Agilent Technologies, LTE Uplink Interference Generation. (2011) User manual

18. Nomor Research GmbH and Rohde \& Schwarz, LTE Uplink Interference Generation. (2011) User manual

19. Tdoc S-10-103 Global Certification Forum, Creation of an End to End Data Performance Measurement Procedure, in GCF Steering Group, \#43 meeting, Germany (June 2010)

20. RP-101422 3GPP Tdoc, Study item proposal: UE Application Layer Data Throughput Performance, in 3GPP TSG-RAN \#50 meeting, Turkey (December 2010)

doi:10.1186/1687-6180-2011-61

Cite this article as: Pauli et al.: Reproducible LTE uplink performance analysis using precomputed interference signals. EURASIP Journal on Advances in Signal Processing 2011 2011:61.

\section{Submit your manuscript to a SpringerOpen ${ }^{\mathcal{O}}$ journal and benefit from:}

- Convenient online submission

- Rigorous peer review

- Immediate publication on acceptance

- Open access: articles freely available online

- High visibility within the field

- Retaining the copyright to your article

Submit your next manuscript at $>$ springeropen.com 